\title{
Effective Purification Procedure of Amaranthin from Amaranthus Celosia argentea inflorescence
}

\author{
Parunya Thiyajai $^{1,2}$, Daichi Kawai ${ }^{1}$, Tomoyuki Koyama ${ }^{1, *}$ \\ ${ }^{1}$ Graduate School of Marine Science and Technology, Tokyo University of Marine Science and Technology, 4-5-7 Konan, Mina- \\ to-ku, Tokyo, 108-8477, Japan. \\ ${ }^{2}$ Institute of Nutrition, Mahidol University, Salaya, Phutthamonthon, Nakhon Pathom, 73170, Thailand.
}

How to cite this paper: Parunya Thiyajai, Daichi Kawai, Tomoyuki Koyama. (2021) Effective Purification Procedure of Amaranthin from Amaranthus Celosia argentea inflorescence. International Journal of Food Science and Agriculture, 5(3), 370-375.

DOI: $10.26855 / \mathrm{ijfsa} .2021 .09 .005$

Received: June 2, 2021

Accepted: June 28, 2021

Published: July 15, 2021

*Corresponding author: Tomoyuki Koyama, Graduate School of Marine Science and Technology, Tokyo University of Marine Science and Technology, 4-5-7 Konan, Minato-ku, Tokyo, 108-8477, Japan.

Email: tskoyama@kaiyodai.ac.jp

\begin{abstract}
In order to develop the utility of amaranthin utility as natural red pigments in the edible plants, the extraction and purification procedures with arrangements were introduced. Freeze-dried amaranthus inflorescence was purposed for extraction using $80 \%$ ethanol after the washed-out step with ethanol to remove coexisting impurities (e.g., polyphenolics and betaxanthins). The purification methods for a similar betacyanin compound, betanin (found in beetroots), were arranged for that of amaranthin in Celosia argentea (CA). The prepared crude extract underwent column chromatography on an ODS column using HPLC system. After purification as a single peak in the chromatogram, the compound was confirmed as amaranthine based on its UV spectrum, MS data and HPLC data. Amaranthine is also expected as natural pigments for food products and as potent antioxidant for health-promoting foods. The reported procedure will effectively enhance the utility of potential ingredients for scientific research and applications for food processing in the future.
\end{abstract}

\section{Keywords}

Amaranthin, Celosia argentea, Antioxidant, LC-MS Analysis, Purification

\section{Introduction}

Amaranthin can be found in common edible and ornamental plants such as amaranth, quinoa, Malabar spinach and Celosia spp. Moreover, red inflorescence of Celosia argentea (CA) containing amaranthin has been suggested as an alternative betalain source for food application [1], but there is not enough informative evidence for application to the food industry as an ingredient. Amaranthin and betanin are two major betacyanin compounds belonging to betalain pigments in plants [2]. Betanin, which is a red pigment inedible beetroot, is widely used as a natural color additive for the food processing industry [3, 4]. It has been approved as one safety ingredients by the European Union and under Section 73.40 in the Title 21 of the Code of Federal Regulations (CFR) by the Food and Drug Administration (FDA) in the United States [5]. Betanin is also apotent antioxidant with various health benefit effects based on numerous scientific research reports $[6,7]$ due to ease of availability as ingredients or as food materials. Alternatively, amaranthin which is abundant in unused food resources, has shown possibilities comparable to betanin. In order to develop the utility and development of amaranthin as food ingredients, or resource of healthy food, the effective purification and the evaluation are required. In this paper, the effective purification procedure for amaranthin and its evaluation using liquid chromatography-mass spectrometry (LCMS) are reported.

\section{Methods}

\subsection{Chemicals}

Cosmosil 75 C18-OPN as reverse phase silica gel for column chromatography was purchased from Nacalai Tesque, INC (Kyoto, Japan). Ascorbic acid, and 2,2'-azobis(2-methylpropionamidine) dihydrochloride (AAPH) were purchased 
from Fujifirm Wako Pure Chemical Industries, Ltd. (Osaka, Japan). 6-hydroxy-2,5,7,8-tetramethylchroman-2-carboxylic acid (trolox), and 2,4,6-tris(2-pyridyl)-s-triazine (TPTZ) were purchased from Tokyo Chemical Industry Co., Ltd. (Tokyo, Japan). All reagents and solvents were of analytical or HPLC grade.

\subsection{Sample preparation}

Fresh red CA inflorescences was purchased from Market in Tokyo, Japan. After rinsing and freeze drying, the inflorescence part of CA were ground into powder and kept at $-20^{\circ} \mathrm{C}$ for further extraction. In the each extraction step, samples were weighed and added pre-cooled solvents at a ratio of 1:100 (sample : solvent). The mixture was vortexed, sonicated, shaken for $10 \mathrm{~min}$, and centrifuged at $2,600 \times g, 4^{\circ} \mathrm{C}$ to separate into supernatant and precipitate. The precipitate was re-extracted 2 more times. Then, the three supernatants were mixed for the next steps. Finally crude extract evaporated and freeze dried. The extracts were re-dissolved with deionized water and kept in freezer for further analysis. Yield (\%) for extraction and separation step was calculated using weight of freeze dried samples. The scheme of extraction and purification steps was summarized in Figure 1. Data of purification steps were explained in the results session as below.

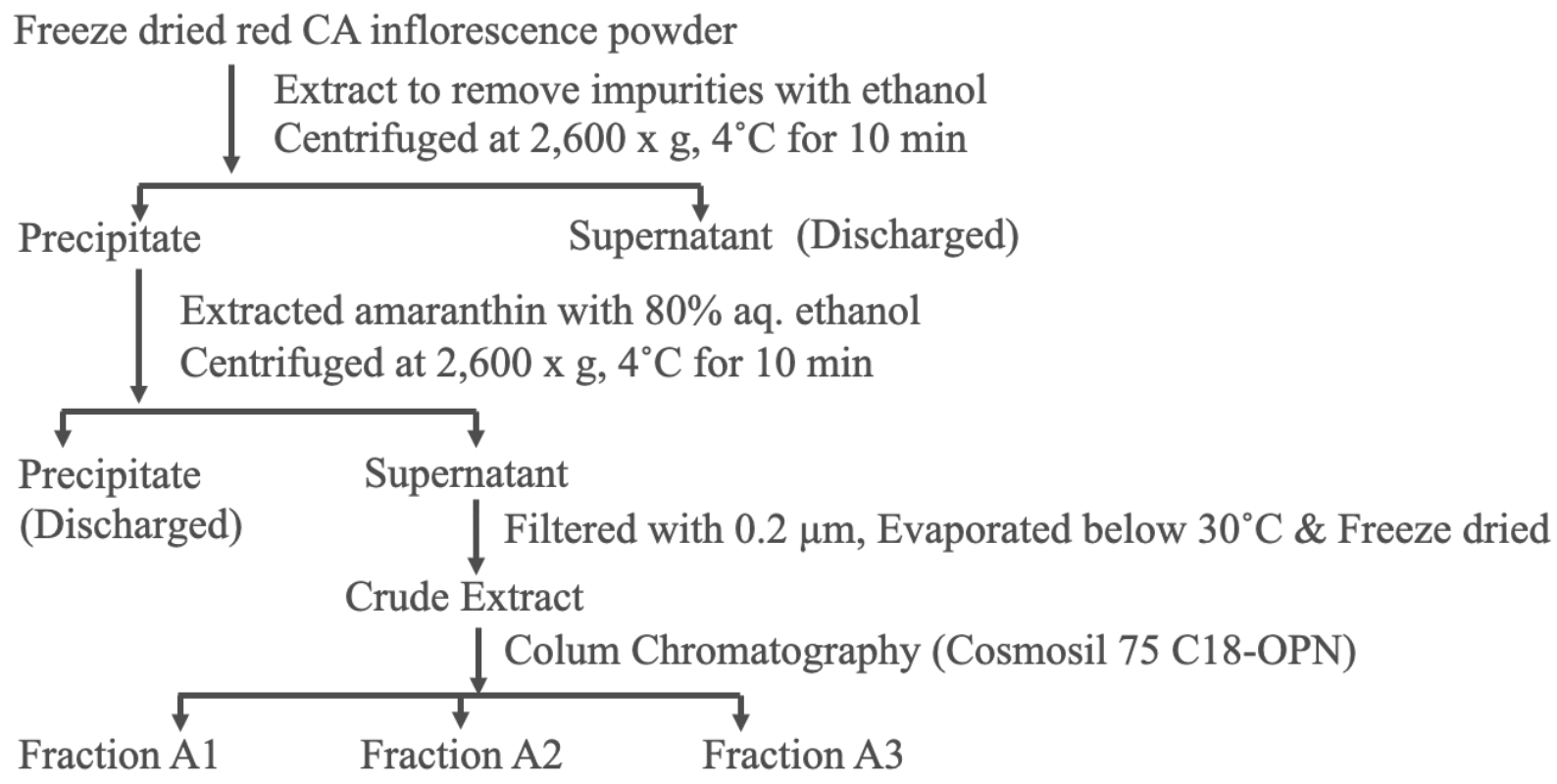

Figure 1. The scheme of extraction and purification of amaranthin.

\subsection{HPLC separation system}

The final purification of amaranthin was carried out on reversed phase silica gel column ODS-HG5 (5 $\mu \mathrm{m}, 20 \times 250$ mm, Nomura chemical, Shizuoka, Japan) using HPLC system (Shimadzu, Kyoto, Japan) consisted of pump, manual injector and UV detector. Mobile phase was $10 \%$ aqueous methanol containing $0.5 \%$ formic acid and $100 \%$ methanol with flow rate at $5.0 \mathrm{~mL} / \mathrm{min}$. The compounds were monitored at $470 \mathrm{~nm}$ or $536 \mathrm{~nm}$ in this step. Data was recorded on PC with integrator RecPro (Runtime Instruments, Tokyo, Japan). Injection volume to the system is below $1 \mathrm{~mL}$ after filtration with $0.2 \mu \mathrm{m}$, the sample was solved in small volume of neutral water and add methanol to adjust polarity to starting mobile phase.

\subsection{Identification of betalains using LCMS}

Identification of betalains was performed using liquid chromatography-mass spectrometry (LCMS) Shimadzu-2010 consisted of degasser, binary pump, autoinjector, column oven, diode array detector and LCMS solution software version 3.41.324 (Shimadzu, Kyoto, Japan). The extracts were separated onto the Devolosil ODS-HG5 column (2.0× 250 $\mathrm{mm}, 5 \mu \mathrm{m}$, Nomura Chemical Co., Ltd., Aichi, Japan) at flow rate of $0.1 \mathrm{~mL} / \mathrm{min}$. Column compartment was set at $40^{\circ} \mathrm{C}$. Mobile phase consisted of mobile phase A (1\% formic acid in water) and B (methanol). Betalains were separated starting from $0-12 \mathrm{~min}, 5-25 \% \mathrm{~B} ; 12-16 \mathrm{~min}, 25-70 \% \mathrm{~B} ; 16-20 \mathrm{~min}, 70-100 \% \mathrm{~B}, 100 \% \mathrm{~B}$ for $5 \mathrm{~min}$, then return to the starting condition for equilibration. The injection volume was $10 \mu \mathrm{L}$. Betacyanins were detected at $535 \mathrm{~nm}$, while betaxanthins were monitored at 462 or $470 \mathrm{~nm}$, respectively. HPLC-DAD was coupled to electrospray ionization (ESI) source 
and LCMS solution software version 3.41.324 operating in the positive ionization mode. The analyses were scanned from $\mathrm{m} / \mathrm{z}$ 50-2000. ESI source was set with a drying gas (nitrogen) flow of $1.5 \mathrm{~L} / \mathrm{min}$. The curved desolvation line $(\mathrm{CDL})$ and heat block temperature was maintained at 250 and $300^{\circ} \mathrm{C}$, respectively. The detector voltage was $1.2 \mathrm{kV}$.

\subsection{Total betacyanin contents}

The appropriate dilution of extracts with water were measured the absorbance at $536 \mathrm{~nm}$ using UV-Visible spectrophotometer (UV-1600 PC, Shimadzu, Japan). The calculation of betacyanin contents was followed Lambert Beer's law using the equation below where " $\mathrm{A}$ " is $\mathrm{OD}_{536} \mathrm{~nm}$ for the maximum absorption of amaranthin and betanin, "V" is the total volume of extract $(\mathrm{mL}), \mathrm{DF}$ is the dilution factor, "MW" is molecular weight of amaranthin (726.6), " $\varepsilon$ " is molecular extinction coefficient of amaranthin $\left(56,600 \mathrm{M}^{-1} \mathrm{~cm}^{-1}\right)$ [8], "L" is the path length of the cuvette $(1 \mathrm{~cm})$ and "W" is the weight of extract (g). Total betacyanin content (mg amaranthin equivalent/mg extract) $=\mathrm{AV}(\mathrm{DF})(\mathrm{MW}) / \varepsilon \mathrm{LW}$.

\subsection{Antioxidant activity (ORAC assay)}

The antioxidant activities of the extracts were evaluated by oxygen radical absorbance capacity (ORAC) assay. Basically, the assay measures the reduction of fluorescence intensity from fluorescent probe caused from radical generated by AAPH [9]. The diluted extracts, ascorbic acid or trolox standard (0-100 $\mu \mathrm{M})(25 \mu \mathrm{L})$ were mixed with $150 \mu \mathrm{L}$ of $81.7 \mathrm{nM}$ fluorescein solution in $75 \mathrm{mM}$ potassium phosphate buffer (pH 7.2). After incubation of mixture for $30 \mathrm{~min}$ at $37^{\circ} \mathrm{C}$ in the dark, $25 \mu \mathrm{L}$ of freshly prepared AAPH solution $153 \mathrm{mM}$ in $75 \mathrm{mM}$ potassium phosphate buffer (pH 7.2) was added for starting the reaction. The fluorescence intensity was read for $1.5 \mathrm{~h}$ every $1 \mathrm{~min}$ using automate plate reader with 96-well plate (SPECTRAFluor Plus, TECAN, Salzburg, Austria). The excitation and emission wavelength was 485 and $535 \mathrm{~nm}$, respectively. The ORAC values were reported as $\mu$ mol Trolox equivalent (TE)/g extract.

\subsection{Statistical analysis}

All experiments were analyzed in triplicates $(\mathrm{n}=3)$ and reported as mean \pm S.D. (standard deviation). One-way analysis of variance (ANOVA) followed by Turkey's multiple range test was used to compare the mean differences among all parameters. Differences were considered to be significant at the $p<0.05$. All statistical analysis was tested using the IBM. SPSS. Statistic version 19 (IBM SPSS, Chicago, IL, USA).

\section{Results}

\subsection{Amaranthin extraction and purification}

Amaranthin was purified from red CA inflorescence (Figure 1) containing a high level of phenolics. Thus, in the first step, the raw materials were extracted with commercially $99.5 \%$ ethanol to partly remove phenolics and betaxanthin possessing orange-yellow color. Then amaranthin was extracted from the residual precipitant with $80 \%$ aqueous ethanolto get crude extract yielded $29.93 \pm 0.59 \%$ with the red-purple color (Table 1 ). The crude extract was separated on a Cosmosil 75 C18-OPN column. The two fractions, A1 and A2, were eluted by water, produced orange-yellow and red-purple colors, respectively. The other fraction displayed an orange-brown color and was eluted by $99.5 \%$ ethanol (Fig. 3). The percent yield of each fraction was $5.30 \pm 0.68 \%, 2.44 \pm 0.52 \%$, and $5.96 \pm 0.32 \%$ for fractions A1, A2, and A3, respectively. The red-purple color is one of a good indicator of a concentrated amaranthin, such as betacyanins. Fraction A2 was further purified on an ODS column using HPLC. The chromatogram at $470 \mathrm{~nm}$ is shown in Figure 2. Fractions A2-2 and A2-3 displayed a purple color for detection at $536 \mathrm{~nm}$. The percent yield of fractions A2-2 and A2-3 was $0.41 \pm 0.07 \%$ and $0.21 \pm 0.15$, respectively.

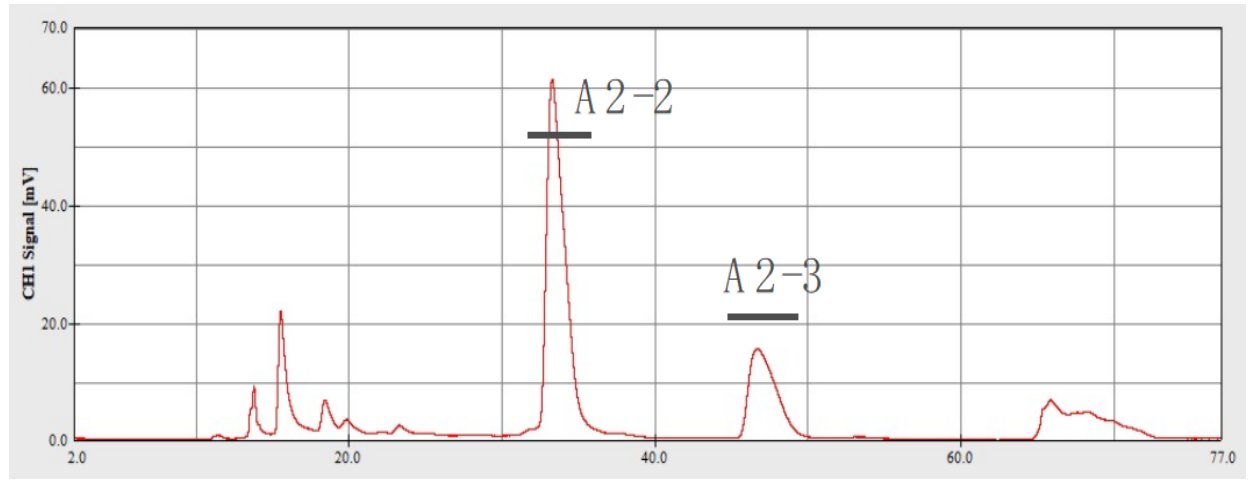

Figure 2. Chromatogram at $470 \mathrm{~nm}$ of fraction A2 extracted from red CA inflorescence. 
Table 1. Yield and color of fraction in purification process of freeze dried CA powder

\begin{tabular}{cccc}
\hline Purification step & Fraction & Yield (\%) & Color \\
\hline Crude extract & - & $29.93 \pm 0.59$ & red-purple \\
Open column & A1 & $5.30 \pm 0.68$ & orange-yellow \\
& A2 & $2.44 \pm 0.52$ & red-purple \\
Preparative column & A3 & $5.96 \pm 0.32$ & orange-brown \\
& A2-2 & $0.41 \pm 0.07$ & red-purple \\
& A2-3 & $0.21 \pm 0.15$ & red-purple \\
\hline
\end{tabular}

Purification step and fraction were described in Figure 1.

Values are mean \pm S.D. $(n=3)$.

\subsection{Analysis using HPLC and MS}

Fractions A2-2 and A2-3 were characterized via LC-MS (Figure 3). The chromatogram at $536 \mathrm{~nm}$, UV-Vis spectrum and mass spectrum (positive mode) of fractions A2-2 and A2-3 are shown here. The single major peak at 14.2 min in the chromatogram of A2-2 had the maximum wavelength at 534-537 nm and molecular ion with $\mathrm{m} / \mathrm{z}=727$. The single prominent peak of fraction A2-3 at $15.3 \mathrm{~min}$ in the chromatogram had amaximum wavelength at $534-537 \mathrm{~nm}$ and $\mathrm{m} / \mathrm{z}=$ 727. These peaks in A2-2 and A2-3 were identified as amaranthin and isoamaranthin, respectively, based on the previous analyzed data [10]. The information indicates that amaranthin was purified from red CA inflorescence at this step. The peak area (\%) of amaranthin and isoamaranthin were $91.28 \pm 5.98$ and $3.21 \pm 0.88$, respectively. Total peak area detected at $470 \mathrm{~nm}$ in the chromatogram with a retention time (RT) of 10-25 min. These data are summarized in Table 2.

Fr. 2-2 (Amaranthin)

DAD
$470 \mathrm{~nm}$

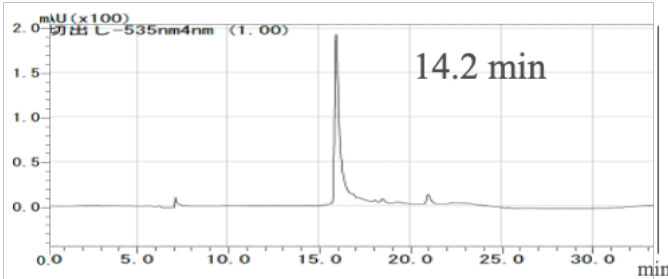

Fr. 2-3 (Isoamaranthin)

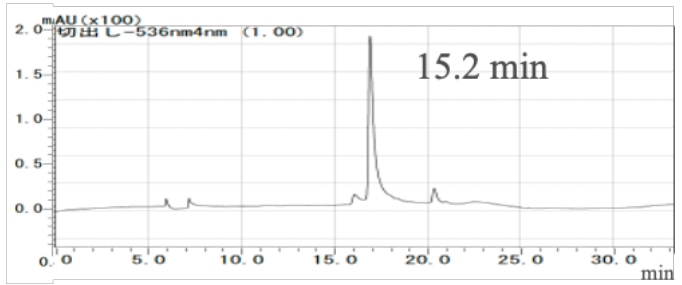

UV
spectrum
at peak
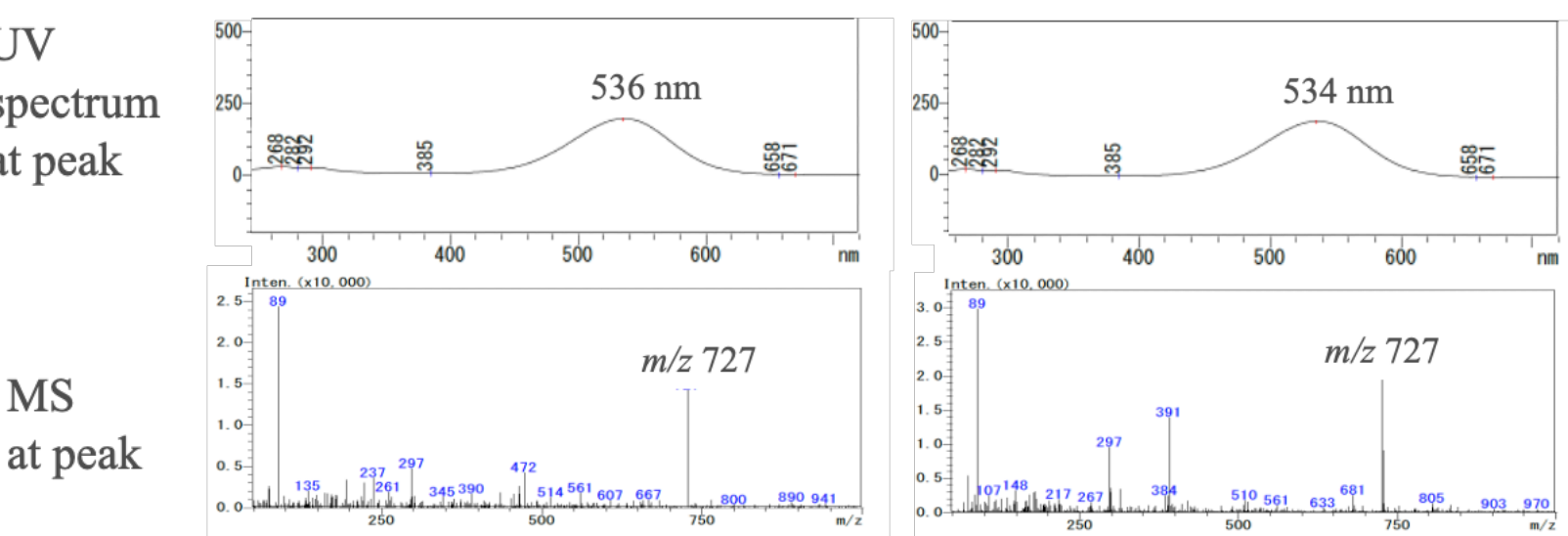

Figure 3. Identification of Fr. 2-2 (amaranthin) and 2-3 (isoamaranthin) purification.

Table 2. Analyzed results of semi purified amaranthins in Fraction A2-2 by HPLC-DAD-MS

\begin{tabular}{ccccccc}
\hline Peak & Compound & Retention time $(\min )$ & $\lambda_{\max }(\mathrm{nm})$ & {$[\mathrm{M}+\mathrm{H}]^{+}$} & Composition in Fr. 2 (\%) & Purity (\%) \\
\hline Fr. A2-2 & Amaranthin & 14.2 & 536 & 727 & $91.28 \pm 5.98$ & 89.8 \\
Fr. A2-3 & Isoamaranthin & 15.2 & 534 & 727 & $3.21 \pm 088$ & 85.1 \\
\hline
\end{tabular}

Composition in Fr. 2 (\%) are based on the peak area in chromatogram of Fr. 2 as shown in Figure 2.

Purity (\%) are based on the peak area in chromatogram in Figure 3. 


\subsection{Evaluating data for CA extract and purified amaranthin}

For betalains purification, $80 \%$ aqueous ethanol is commonly used because of its ability to precipitate proteins and other insoluble products. In our trial experiment, 99.5\% ethanol was unsuccessful in extracting amaranthin from CA extract; the red color remained in the precipitant perfectly. In contrast, water can easily extract the highly polar pigments with red-colored supernatant. Subsequently, total betacyanin contents (TBC) and antioxidant activities (ORAC) were compared between extracts prepared from the same CA powder stock. The water extract showed $12.3 \pm 4.3 \mathrm{mg} / \mathrm{g}$ extract (1.2\% shared) for TBC and 984.3 $\pm 148.5 \mu \mathrm{mol}$ TE/g extract for ORAC. Furthermore, $80 \%$ aqueous ethanol extract showed $9.1 \pm 2.0 \mathrm{mg} / \mathrm{g}$ extract (shared $0.9 \%$ ) for TBC and 3,021.64 $\pm 209.74 \mu \mathrm{mol} \mathrm{TE} / \mathrm{g}$ extract for ORAC. The $80 \%$ ethanol could not completely extract amaranthin; possibly, phenolics, as impurities could be extracted better. Alternatively, the purified amaranthin as Fr. A2-2 showed 25.7 $\pm 2.1 \mu \mathrm{mol}$ TE/ $\mu \mathrm{mol}$ in the ORAC assay. It was stronger than the ORAC value of ascorbic acid, as the positive control was $17558 \pm 102.6 \mu \mathrm{mol} \mathrm{TE} / \mathrm{g}$ extract). Based on further analysis using LCMS data, there are no impurities such as polyphenolics ( $\lambda_{\max }: 254$ and $350 \mathrm{~nm}$; RT: $\left.24.42 \mathrm{~min}\right)$, dopanine $\left(\lambda_{\max }\right.$ : $274 \mathrm{~nm}$; RT: $7.30 \mathrm{~min}$ ) and betaxanthins $\left(\lambda_{\max }: 475 \mathrm{~nm}\right.$; RT: 20.1 and $\left.22.6 \mathrm{~min}\right)$, existing in $80 \%$ ethanol extract were not detected in the purified amaranthin.

\section{Discussion}

Amaranthin and betaninhave common aglycon in their structures. The sugar component is glucuronyl glucose and glucose for amaranthin and betanin, respectively. Their chemical properties are also similar, but the polarity of amaranthin is higher than betanin $[2,11]$. This means that some physiological activities will differ from each other. In this report, the differences about polarity could contribute to the effective extraction and purification of amaranthin from red CA. The method for amaranthin and betanin extraction was similar. In actuality, CA contained a higher amount of phenolics [10]. In order to remove these impurities, a 99.5\% ethanol extraction was effective. In terms of purification, $80 \%$ methanol instead of ethanol was used for protein and insoluble compound precipitation. After the extraction, the crude extracts were applied to the open column using reverse phase silica gel and HPLC system. The mobile phase for separating amaranthin and betanin was different due to the polarity of the compounds. Amaranthin was separated using $10 \%$ methanol in water containing $0.5 \%$ formic acid, whereas betanin was separated by $15 \%$ methanol in water containing $0.5 \%$ formic acid. The $\%$ yield for purified amaranthin and betanin was $0.41 \%$ and $0.63 \%$, respectively. The purity, based on peak area identified by LCMS, was approximately $91.28 \%$ and $95.10 \%$ for amaranthin and betanin, respectively.

The extraction and purification of both compounds included three main steps: (1) extraction, (2) separation on ODS gel in a glass column, and (3) separation by preparative column. Based on our unpublished data, the percent yield of amaranthin $(0.41 \pm 0.07 \%)$ was similar to that of betanin $0.63 \pm 0.09 \%$. Moreover, the CA extract contained a higher amount of phenolics than BV extract. Amaranthin was not extracted with 99.5\% ethanol, but it was extracted partly with $100 \%$ methanol. The polar index of water, methanol, and ethanol were reported as 9.0, 6.6, and 5.2, respectively [12]. In order to remove impurities from the CA extract, ethanol proved a better choice due to its polarity. Major impurities in CA amaranthin include polyphenolics, not sucrose; this is in contract to betanin found in beetroot. So the procedure was suitable for amaranthin extraction and purification from CA extract.

\section{Conclusion}

In this report, a simple technique for obtaining an interesting natural pigment, amaranthin, from red CA inflorescences was introduced with analyzed data. After the extraction, the crude extracts were separated on ODS gel to obtain the amaranthin containing fraction. The red-purple fraction was further separated using a preparative ODS column via HPLC. The optimal mobile phase for the separation of amaranthin and betanin was different due to the different polarities of the compounds. Amaranthin was separated using $10 \%$ methanol in water containing $0.5 \%$ formic acid. The yield for purified amaranthin was $0.41 \%$. The purity, based on the peak area identified by LCMS, was $91.28 \%$ for amaranthin. The chemical properties of amaranthin are similar to those of betanin. However, CA contained a higher amount of phenolics; thus, the phenolic extraction using 99.5\% ethanol was effective. In terms of purification, ethanol treatment before extraction and optimal separating conditions were used to remove impurities during the procedure. The reported procedure will effectively contribute to obtaining amaranthin for use in laboratories and trials for food processing.

This study sheds light on the potency and utilization of amaranthin in the extract and purified compound forms, which can be applied as a natural colors, functional ingredients or nutraceuticals in the future.

\section{Acknowledgements}

The authors would like to thank the Ministry of Education, Culture, Sports, Science and Technology (MEXT) for the financial support. 


\section{References}

[1] Schliemann, W., Cai, Y., Degenkolb, T., Schmidt, J., and Corke, H. (2001). Betalains of Celosia argentea. Phytochemistry, 58, 159-165.

[2] Sawicki, T., Topolska, J., Romaszko, E., and Wiczkowski, W. (2018). Profile and Content of Betalains in Plasma and Urine of Volunteers after Long-Term Exposure to Fermented Red Beet Juice. Journal of Agricultural and Food Chemistry, 66, 4155-4163.

[3] Polturak, G. and Aharoni, A. (2018). 'La Vie En Rose’: Biosynthesis, Sources, and Applications of Betalain Pigments. Molecular Plant, 11, 7-22. https://doi.org/10.1016/j.molp.2017.10.008.

[4] Rodriguez-Amaya, D. B. (2016). Natural Food Pigments and Colorants. Current Opinion in Food Science, 7, $20-26$. http://dx.doi.org/10.1016/j.cofs.2015.08.004.

[5] Khan, M. I. (2016). Plant Betalains: Safety, Antioxidant Activity, Clinical Efficacy, and Bioavailability. Comprehensive Reviews in Food Science and Food Safety, 15, 316-330.

[6] Slimen, I. B., Najar, T., and Abderrabba, M. (2017). Chemical and Antioxidant Properties of Betalains. Journal of Agricultural and Food Chemistry, 65, 675-689.

[7] Azeredo, H. M. C. (2009). Betalains: Properties, Sources, Applications, and Stability—A Review. International Journal of Food Science and Technology, 44, 2365-2376.

[8] Cai, Y. and Sun, M. (2005). HPLC Characterization of Betalains from Plants in the Amaranthaceae. Journal of Chromatographic Science, 43, 454-460.

[9] Prior, L. R., Wu, X., and Schaich, K. (2005). Standardized Methods for the Determination of Antioxidant Capacity and Phenolics in Foods and Dietary Supplements. Journal of Agricultural and Food Chemistry, 53, 4290-4302.

[10] Tiyajai, P. and Koyama, T. (2021). Binary Ethanol-Water Solvents Affect Betalain Contents and Health-Promoting Properties of Red Celosia argentea Inflorescence Extracts. International Food Research Journal (in press).

[11] Cai, Y., Sun, M., and Corke, H. (2003). Antioxidant Activity of Betalains from Plants of the Amaranthaceae. Journal of Agricultural and Food Chemistry, 51, 2288-2294.

[12] Snyder, L. R. (1974). Classification of the Solvent Properties of Common Liquids. Journal of Chromatography, 92, $233-240$. 